\title{
Measurement of the $\bar{\Lambda}$ Polarization in $\nu_{\mu}$ Charged Current Interactions in the NOMAD Experiment
}

\author{
NOMAD Collaboration
}

P. Astier ${ }^{n}$ D. Autiero ${ }^{\text {h }}$ A. Baldisseri ${ }^{r}$ M. Baldo-Ceolin ${ }^{m}$ M. Banner ${ }^{n}$ G. Bassompierre ${ }^{\text {a }}$ N. Besson ${ }^{r}$ I. Bird ${ }^{\text {h,i }}$ B. Blumenfeld ${ }^{b}$ F. Bobisut ${ }^{m}$ J. Bouchez ${ }^{r}$ S. Boyd ${ }^{t}$ A. Bueno ${ }^{c, x}$ S. Bunyatov ${ }^{f}$ L. Camilleri ${ }^{\text {h A. Cardini }}{ }^{\text {j P.W. Cattaneo }}{ }^{o}$ V. Cavasinni ${ }^{\mathrm{p}}$ A. Cervera-Villanueva ${ }^{\mathrm{h}, \mathrm{v}}$ A. Chukanov ${ }^{\mathrm{f}}$ G. Collazuol ${ }^{\mathrm{m}}$ G. Conforto ${ }^{\mathrm{h}, \mathrm{u}}$ C. Conta $^{\mathrm{o}}$ M. Contalbrigo ${ }^{\mathrm{m}}$ R. Cousins ${ }^{j}$ D. Daniels ${ }^{c}$ H. Degaudenzi ${ }^{i}$ T. Del Prete ${ }^{p}$ A. De Santo ${ }^{h}$ T. Dignan ${ }^{c}$ L. Di Lella ${ }^{\text {h E. do Couto e Silva }}{ }^{\mathrm{h}}$ J. Dumarchez ${ }^{n}$ M. Ellis ${ }^{\text {t }}$ T. Fazio ${ }^{a}$ G.J. Feldman ${ }^{\text {c }}$ R. Ferrari ${ }^{\circ}$ D. Ferrère ${ }^{\mathrm{h}}$ V. Flaminio ${ }^{\mathrm{p}}$ M. Fraternali ${ }^{\mathrm{o}}$ J.-M. Gaillard $^{\mathrm{a}}$ E. Gangler ${ }^{\mathrm{h}, \mathrm{n}}$ A. Geiser ${ }^{\mathrm{e}, \mathrm{h}}$ D. Geppert ${ }^{\mathrm{e}}$ D. Gibin ${ }^{\mathrm{m}}$ S. Gninenko ${ }^{\mathrm{h}, \ell}$ A. Godley ${ }^{\mathrm{t}}$ J.-J. Gomez-Cadenas ${ }^{\mathrm{h}, \mathrm{v}}$ J. Gosset $^{\mathrm{r}}$ C. Gößling e M. Gouanère ${ }^{\mathrm{a}}$ A. Grant ${ }^{\mathrm{h}}$ G. Graziani ${ }^{\mathrm{g}}$ A. Guglielmi ${ }^{\mathrm{m}}$ C. Hagner ${ }^{\mathrm{r}}$ J. Hernando $^{\mathrm{v}}$ D. Hubbard ${ }^{\mathrm{c}}$ P. Hurst ${ }^{c}$ N. Hyett ${ }^{k}$ E. Iacopini ${ }^{g}$ C. Joseph ${ }^{i}$ F. Juget ${ }^{i}$ M. Kirsanov ${ }^{\ell}$ O. Klimov ${ }^{f}{ }^{\text {J. Kokkonen }}{ }^{\text {h }}$ A. Kovzelev ${ }^{\ell, o}$ A. Krasnoperov a,f D. Kustov ${ }^{\mathrm{f}}$ V. Kuznetsov ${ }^{\text {f,h }}$ S. Lacaprara ${ }^{\mathrm{m}}$ C. Lachaud ${ }^{\mathrm{n}}$ B. Lakić ${ }^{\mathrm{w}}$ A. Lanza ${ }^{\circ}$ L. La Rotonda ${ }^{\mathrm{d}}$ M. Laveder ${ }^{m}$ A. Letessier-Selvon ${ }^{n}$ J.-M. Levy ${ }^{n}$ L. Linssen ${ }^{\mathrm{h}}$ A. Ljubičić ${ }^{\mathrm{w}}$ J. Long ${ }^{\mathrm{b}}$ A. Lupi ${ }^{\mathrm{g}}$ A. Marchionni ${ }^{\mathrm{g}}$ F. Martelli ${ }^{\mathrm{u}}$ X. Méchain ${ }^{r}$ J.-P. Mendiburu ${ }^{a}$ J.-P. Meyer ${ }^{r}$ M. Mezzetto ${ }^{m}$ S.R. Mishra ${ }^{\text {c,s }}$ G.F. Moorhead ${ }^{\mathrm{k}}$ D. Naumov ${ }^{\mathrm{f}}$ P. Nédélec ${ }^{\mathrm{a}}$ Yu. Nefedov ${ }^{f}$ C. Nguyen-Mau ${ }^{i}$ D. Orestano ${ }^{q}$ F. Pastore ${ }^{q}$ L.S. Peak ${ }^{t}$ E. Pennacchio ${ }^{u}$ H. Pessard ${ }^{a}$ R. Petti ${ }^{\text {h,o }}$ A. Placci ${ }^{h}$ G. Polesello ${ }^{\circ}$ D. Pollmann ${ }^{\text {e }}$ A. Polyarush ${ }^{\ell}$ B. Popov ${ }^{\text {f,n }}$ C. Poulsen ${ }^{k}$ J. Rico ${ }^{x}$ P. Riemann ${ }^{\mathrm{e}}$ C. Roda ${ }^{\mathrm{h}, \mathrm{p}}$ A. Rubbia ${ }^{\mathrm{h}, \mathrm{x}}$

F. Salvatore ${ }^{o}$ K. Schahmaneche ${ }^{n}$ B. Schmidt ${ }^{e, h}$ T. Schmidt ${ }^{e}$ 
M. Sevior ${ }^{k}$ D. Sillou ${ }^{a}$ F.J.P. Soler ${ }^{\text {h,t }}$ G. Sozzi ${ }^{i}$ D. Steele ${ }^{b, i}$ U. Stiegler ${ }^{\text {h }}$ M. Stipčević ${ }^{w}$ Th. Stolarczyk ${ }^{r}$ M. Tareb-Reyes ${ }^{i}$ G.N. Taylor ${ }^{\mathrm{k}}$ V. Tereshchenko ${ }^{\mathrm{f}}$ A. Toropin ${ }^{\ell}$ A.-M. Touchard ${ }^{\mathrm{n}}$ S.N. Tovey ${ }^{\mathrm{h}, \mathrm{k}}$ M.-T. Tran ${ }^{\mathrm{i}}$ E. Tsesmelis ${ }^{\mathrm{h}}$ J. Ulrichs ${ }^{\mathrm{t}}$ L. Vacavant ${ }^{\mathrm{i}}$ M. Valdata-Nappi ${ }^{\mathrm{d}, 1}$ V. Valuev ${ }^{\mathrm{f}, \mathrm{j}}$ F. Vannucci ${ }^{\mathrm{n}}$ K.E. Varvell ${ }^{\mathrm{t}}$ M. Veltri ${ }^{\mathrm{u}}$ V. Vercesi ${ }^{\mathrm{O}}$ G. Vidal-Sitjes ${ }^{\mathrm{h}}$ J.-M. Vieira ${ }^{i}$ T. Vinogradova ${ }^{j}$ F.V. Weber ${ }^{c, h}$ T. Weisse ${ }^{e}$ F.F. Wilson ${ }^{\text {h }}$ L.J. Winton ${ }^{k}$ B.D. Yabsley ${ }^{t}$ H. Zaccone ${ }^{\mathrm{r}}$ K. Zuber ${ }^{\mathrm{e}}$ P. Zuccon ${ }^{\mathrm{m}}$

${ }^{\mathrm{a}}$ LAPP, Annecy, France

${ }^{\mathrm{b}}$ Johns Hopkins Univ., Baltimore, MD, USA

${ }^{\mathrm{c}}$ Harvard Univ., Cambridge, MA, USA

${ }^{\mathrm{d}}$ Univ. of Calabria and INFN, Cosenza, Italy

e Dortmund Univ., Dortmund, Germany ${ }^{\mathrm{f}}$ JINR, Dubna, Russia

${ }^{\mathrm{g}}$ Univ. of Florence and INFN, Florence, Italy

${ }^{\mathrm{h}}$ CERN, Geneva, Switzerland

${ }^{\mathrm{i}}$ University of Lausanne, Lausanne, Switzerland

${ }^{j} U C L A$, Los Angeles, CA, USA

${ }^{\mathrm{k}}$ University of Melbourne, Melbourne, Australia

${ }^{\ell}$ Inst. Nucl. Research, INR Moscow, Russia

${ }^{\mathrm{m}}$ Univ. of Padova and INFN, Padova, Italy

${ }^{\mathrm{n}}$ LPNHE, Univ. of Paris VI and VII, Paris, France

${ }^{\circ}$ Univ. of Pavia and INFN, Pavia, Italy

${ }^{\mathrm{p}}$ Univ. of Pisa and INFN, Pisa, Italy

${ }^{\mathrm{q}}$ Roma Tre University and INFN, Rome, Italy

${ }^{\mathrm{r}} D A P N I A, C E A$ Saclay, France

${ }^{\mathrm{s}}$ Univ. of South Carolina, Columbia, SC, USA

${ }^{\mathrm{t}}$ Univ. of Sydney, Sydney, Australia

${ }^{\mathrm{u}}$ Univ. of Urbino, Urbino, and INFN Florence, Italy

${ }^{v}$ IFIC, Valencia, Spain

${ }^{\mathrm{w}}$ Rudjer Bošković Institute, Zagreb, Croatia

${ }^{x}$ ETH Zürich, Zürich, Switzerland 


\begin{abstract}
We present a measurement of the polarization of $\bar{\Lambda}$ hyperons produced in $\nu_{\mu}$ charged current interactions. The full data sample from the NOMAD experiment has been analyzed using the same $V^{0}$ identification procedure and analysis method reported in a previous paper [1] for the case of $\Lambda$ hyperons. The $\bar{\Lambda}$ polarization has been measured for the first time in a neutrino experiment. The polarization vector is found to be compatible with zero.
\end{abstract}

Key words: neutrino interactions, antilambda polarization, nucleon spin, spin transfer

\title{
1 Introduction
}

The spin structure of hadrons has been extensively studied both experimentally and theoretically over the past two decades. Measuring parton spin distributions in different octet baryons can shed light on many phenomena in non-perturbative QCD, such as $S U(3)$ symmetry breaking, baryon spin content, flavor asymmetry in the baryon sea, which were evoked in connection with the proton spin puzzle [2]. Despite some achievements in this field, our current knowledge of the nucleon sea-quark spin distributions and the spin content of the other hyperons is still very poor. One way to learn about the quark spin distributions of unstable hadrons is to measure the polarized quark fragmentation function $\Delta D_{q}^{h}(z)$ of the quark $q$ into the hadron $h$, where $z$ is the fraction of the total hadronic energy carried by the hadron in the laboratory system.

Among various hadrons produced in deep inelastic scattering (DIS) $\Lambda$ and $\bar{\Lambda}$ hyperons are unique because of their parity violating decays into a pair of charged hadrons $\left(\Lambda \rightarrow \mathrm{p} \pi^{-}\right.$and $\bar{\Lambda} \rightarrow \overline{\mathrm{p}} \pi^{+}$respectively) which can be efficiently reconstructed and identified.

The $\Lambda(\bar{\Lambda})$ polarization is measured by the asymmetry in the angular distribution of the protons (antiprotons) or pions in the parity violating decay process $\Lambda \rightarrow \mathrm{p} \pi^{-}\left(\bar{\Lambda} \rightarrow \overline{\mathrm{p}} \pi^{+}\right)$. In the $\Lambda(\bar{\Lambda})$ rest frame the angular distribution of the decay protons (pions) is given by:

$$
\frac{1}{N} \frac{\mathrm{d} N}{\mathrm{~d} \Omega}=\frac{1}{4 \pi}(1+\alpha \mathbf{P} \cdot \mathbf{k})
$$

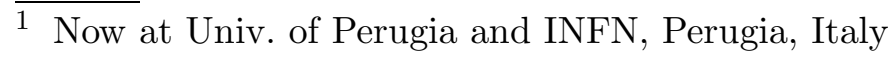


where $\mathbf{P}$ is the $\Lambda(\bar{\Lambda})$ polarization vector, $\alpha=0.642 \pm 0.013[3]$ is the decay asymmetry parameter and $\mathbf{k}$ is the unit vector along the direction of the outgoing positive decay particle (the proton in case of $\Lambda$ and the $\pi^{+}$in case of $\bar{\Lambda})$.

To probe the polarized quark distribution in a hadron $\Delta q_{h}\left(x_{B j}\right)$, where $x_{B j}$ is the standard Bjorken variable, a source of polarized quarks is required. Of the many means of obtaining polarized quarks [4,5], neutrino and antineutrino DIS are exceptional due to their $100 \%$ natural polarization. Moreover, weak interactions provide a source of polarized quarks of specific flavour, which makes the measurement of $\Lambda$ and $\bar{\Lambda}$ polarizations in (anti)neutrino DIS an ideal tool to investigate different spin transfer mechanisms and to check various models of the baryon spin content.

Different physical mechanisms are responsible for the $\Lambda$ and $\bar{\Lambda}$ polarization in the fragmentation regions defined by positive and negative values of $x_{F}=$ $2 p_{L}^{*} / W$. A polarization of the strange sea in the nucleon [6] can manifest itself through a polarization of $\Lambda$ and $\bar{\Lambda}$ hyperons produced in the target fragmentation region $\left(x_{F}<0\right)$ in (anti)neutrino DIS process. Measurements of the polarization of the $\Lambda$ and $\bar{\Lambda}$ hyperons produced in the current fragmentation region $\left(x_{F}>0\right)$ in (anti)neutrino DIS can provide information on the polarized fragmentation functions of quarks and antiquarks into a given hyperon ( $h=\Lambda$ or $\bar{\Lambda}$ ) [7-9]. The longitudinal polarization of a hyperon $h$ produced in neutrino DIS in the current fragmentation region, assuming no polarization transfer from the other (anti)quarks fragmenting into this hyperon, is given by:

$$
P_{\nu}^{h}=-\frac{\left[d\left(x_{B j}\right)+\omega s\left(x_{B j}\right)\right] \Delta D_{u}^{h}(z)-\left(1-y_{B j}\right)^{2} \bar{u}\left(x_{B j}\right)\left[\Delta D_{\bar{d}}^{h}(z)+\omega \Delta D_{\bar{s}}^{h}(z)\right]}{\left[d\left(x_{B j}\right)+\omega s\left(x_{B j}\right)\right] D_{u}^{h}(z)+\left(1-y_{B j}\right)^{2} \bar{u}\left(x_{B j}\right)\left[D_{\bar{d}}^{h}(z)+\omega D_{\bar{s}}^{h}(z)\right]},
$$

where $\omega=\tan ^{2} \theta_{C}\left(\theta_{C}\right.$ is the Cabibbo angle). A measurement of the $\Lambda$ polarization in the current fragmentation region in $\nu_{\mu}$ charged current (CC) DIS provides an estimate of the spin transfer coefficient $C_{u}^{\Lambda}=\Delta D_{u}^{\Lambda}(z) / D_{u}^{\Lambda}(z)$ because of the dominant contribution from the first term in both the numerator and denominator of Eq. (2). However an interpretation of the $\bar{\Lambda}$ polarization in the current fragmentation region in $\nu_{\mu}$ CC DIS is more complicated since the two terms in both the numerator and denominator of Eq. (2) could be of comparable size.

In order to reduce the number of independent fragmentation functions the authors of [7] made the following assumptions:

$$
D_{q}^{\Lambda}(z)=D_{u}^{\Lambda}(z)=D_{d}^{\Lambda}(z)=D_{\bar{u}}^{\bar{\Lambda}}(z)=D_{\bar{d}}^{\bar{\Lambda}}(z)
$$


and

$$
\Delta D_{q}^{\Lambda}(z)=\Delta D_{u}^{\Lambda}(z)=\Delta D_{d}^{\Lambda}(z)=\Delta D_{\bar{u}}^{\bar{\Lambda}}(z)=\Delta D_{\bar{d}}^{\bar{\Lambda}}(z)
$$

The relations (3) and (4) simplify the interpretation of the results of the $\Lambda$ and $\bar{\Lambda}$ polarization measurements in (anti)neutrino DIS. However, these relations may not be valid if a sizable fraction of $\Lambda$ and $\bar{\Lambda}$ hyperons is produced via resonance or heavier hyperon decays.

The NOMAD experiment [10] has collected $1.3 \times 10^{6} \nu_{\mu}$ CC events and has observed an unprecedented number of $\Lambda$ and $\bar{\Lambda}$ decays. A detailed paper devoted to the $\Lambda$ polarization measurement in the NOMAD experiment has been published recently [1]. We present here the first measurement of the polarization of $\bar{\Lambda}$ hyperons produced in $\nu_{\mu}$ CC interactions.

\section{Event selection and $V^{0}$ identification}

The measurement of charged tracks produced in neutrino interactions in the NOMAD detector is performed with a set of drift chambers [11] located inside a dipole magnet with a field of 0.4 Tesla.

A $\bar{\Lambda}$ decay appears in the detector as a $V^{0}$-like vertex: two tracks of opposite charge emerging from a common vertex separated from the primary neutrino interaction vertex (see Fig. 1). A $V^{0}$-like signature is expected also for $\Lambda$ and $\mathrm{K}_{\mathrm{s}}^{0}$ decays and for photon conversions.

The data are compared to the results of a Monte Carlo (MC) simulation based on LEPTO 6.1 [12] and JETSET 7.4 [13] generators for neutrino interactions and on a GEANT [14] based program for the detector response. In our MC simulation $\bar{\Lambda}$ hyperons are not polarized.

The selection procedure for the $\nu_{\mu} \mathrm{CC}$ event sample used in this analysis has been described in [1].

Since the NOMAD detector is unable to distinguish (anti)protons from pions in the momentum range relevant to this analysis, our $V^{0}$ identification procedure relies on the kinematic properties of a $V^{0}$ decay.

For the $V^{0}$ identification a kinematic fit method has been used as described in reference [1]. This fit has been performed for three decay hypotheses: $\mathrm{K}_{\mathrm{s}}^{0} \rightarrow \pi^{+} \pi^{-}, \Lambda \rightarrow \mathrm{p} \pi^{-}, \bar{\Lambda} \rightarrow \overline{\mathrm{p}} \pi^{+}$and for the hypothesis of a photon conversion $\gamma \rightarrow \mathrm{e}^{+} \mathrm{e}^{-}$. The output of the kinematic fits applied to a given $V^{0}$ vertex consists of four $\chi_{V^{0}}^{2}$. The different regions in the four-dimensional $\chi_{V^{0}}^{2}$ space populated by particles identified as $\Lambda, \bar{\Lambda}$ and $\mathrm{K}_{\mathrm{s}}^{0}$ have been selected. 


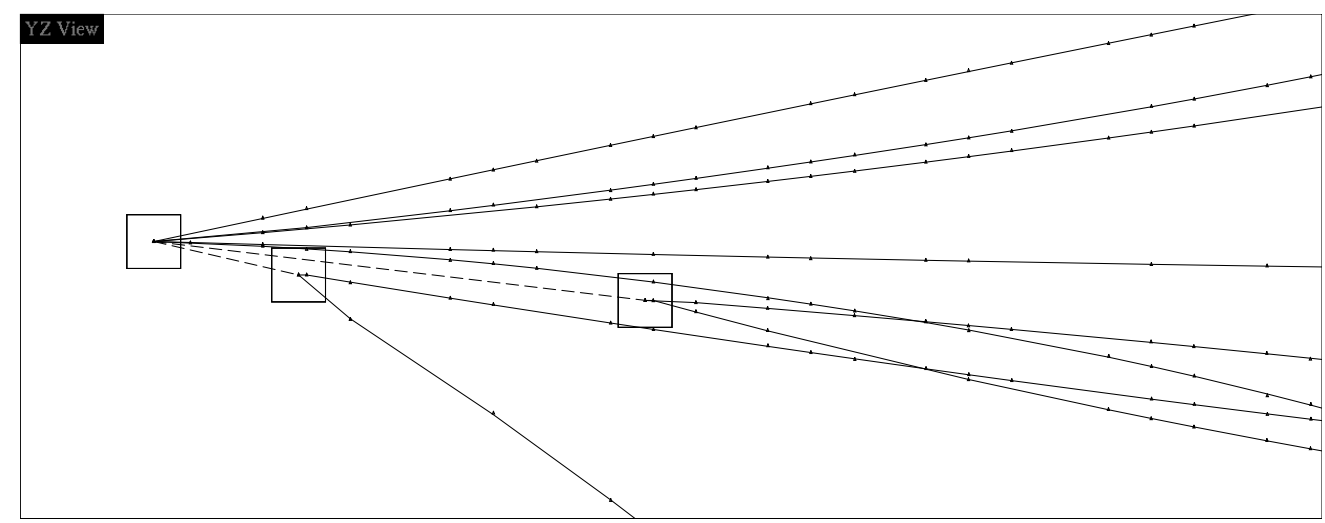

Fig. 1. A reconstructed data event containing $2 V^{0}$ vertices identified as $\Lambda$ and $\bar{\Lambda}$ decays by our identification procedure. The scale on this plot is given by the size of the vertex boxes $\left(3 \times 3 \mathrm{~cm}^{2}\right)$.

Identified $V^{0}$ 's are of two types:

- uniquely identified $V^{0}$ s, which, in the four-dimensional $\chi_{V^{0}}^{2}$ space described above, populate regions corresponding to the decay of different particles;

- ambiguously identified $V^{0}$ s, which populate overlapping kinematic regions where the decays of different particles are simultaneously present.

The treatment of ambiguities aims at selecting a given $V^{0}$ decay with the highest efficiency and the lowest background contamination from other $V^{0}$ types. Our identification strategy consists of two steps:

1) we select a sample of uniquely identified $\bar{\Lambda}$ hyperons which has a purity of $91 \%$

2) we allow ourselves an additional $7 \%$ contribution from a subsample of ambiguously identified $\bar{\Lambda}$ particles resolving the ambiguities between $\bar{\Lambda}$ and $\mathrm{K}_{\mathrm{s}}^{0}$ in favour of maximal purity.

This approach provides an optimum compromise between high statistics and well understood background contamination.

A MC simulation program has been used to study the purity of the overall $\bar{\Lambda}$ sample obtained by our selection criteria in $\nu_{\mu} \mathrm{CC}$ interactions. This study indicates that $89.6 \%$ of the selected $V^{0}$ 's are true $\bar{\Lambda}$ hyperons, $5.4 \%$ are misidentified $\mathrm{K}_{\mathrm{s}}^{0}$ mesons and $5.0 \%$ are due to random track associations. The global reconstruction and identification efficiency is $18.6 \%$. A total of 649 identified $\bar{\Lambda}$ decays is found in our data, representing a significantly larger number than in previous (anti)neutrino experiments performed with bubble chambers [15-19]. Fig. 2 shows the invariant mass distributions for $\bar{p} \pi^{+}$com- 
binations before and after the $V^{0}$ identification procedure.
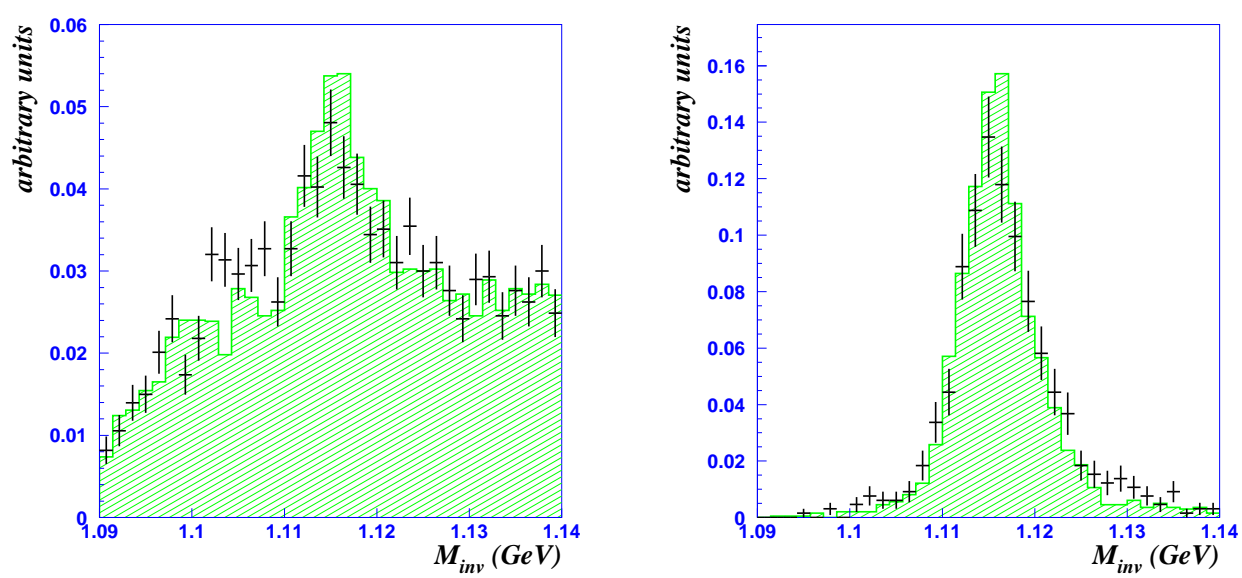

Fig. 2. Normalized invariant mass distributions in both data (points with error bars) and $M C$ (histogram) calculated for $V^{0}$ vertices under the assumption of a $\bar{\Lambda}$ decay before (left) and after (right) the $V^{0}$ identification procedure.

We have compared the distributions of many kinematical variables, describing the neutrino interaction $\left(E_{\nu}, x_{B j}, y_{B j}\right), V^{0}$ production and decay $\left(p_{V}, p_{+}, p_{T}^{i n t}\right)$, $V^{0}$ behaviour in the hadronic jet $\left(p_{T}, x_{F}, z\right)$, obtained in data and MC events. The comparison (see Fig. 3) shows that the experimental distributions of all these kinematical variables are in agreement with the MC results. However, we note that the $x_{F}$ distribution is shifted towards positive values in the data (see Fig. 3e), while it is centered around zero in the MC. A small discrepancy between data and MC may be noticed in the $z$ distribution (Fig. 3i).

\section{Polarization analysis}

For the polarization analysis described below we use the "J" reference system, in which the axes are defined as follows (in the $\bar{\Lambda}$ rest frame):

- the $\mathbf{n}_{x}$ axis is chosen along the reconstructed $W$-boson direction $\left(\vec{e}_{W}\right)$;

- the $\mathbf{n}_{y}$ axis is orthogonal to the $\bar{\Lambda}$ production plane (defined as the plane containing both the target nucleon $\left(\vec{e}_{T}\right)$ and the $W$-boson vectors):

$\mathbf{n}_{y}=\vec{e}_{W} \times \vec{e}_{T} /\left|\vec{e}_{W} \times \vec{e}_{T}\right|$.

- the $\mathbf{n}_{z}$ axis is chosen to form a right-handed coordinate system:

$\mathbf{n}_{z}=\mathbf{n}_{x} \times \mathbf{n}_{y}$.

The experimental resolution for the reconstructed $\cos \theta_{i}=\mathbf{n}_{i} \cdot \mathbf{k}$, where $\mathbf{k}$ is the unit vector in the direction of the outgoing positive track $\left(\pi^{+}\right)$, is found to be about 0.07 . The raw $\cos \theta_{i}$ distributions of $\bar{\Lambda}$ hyperons are affected by 


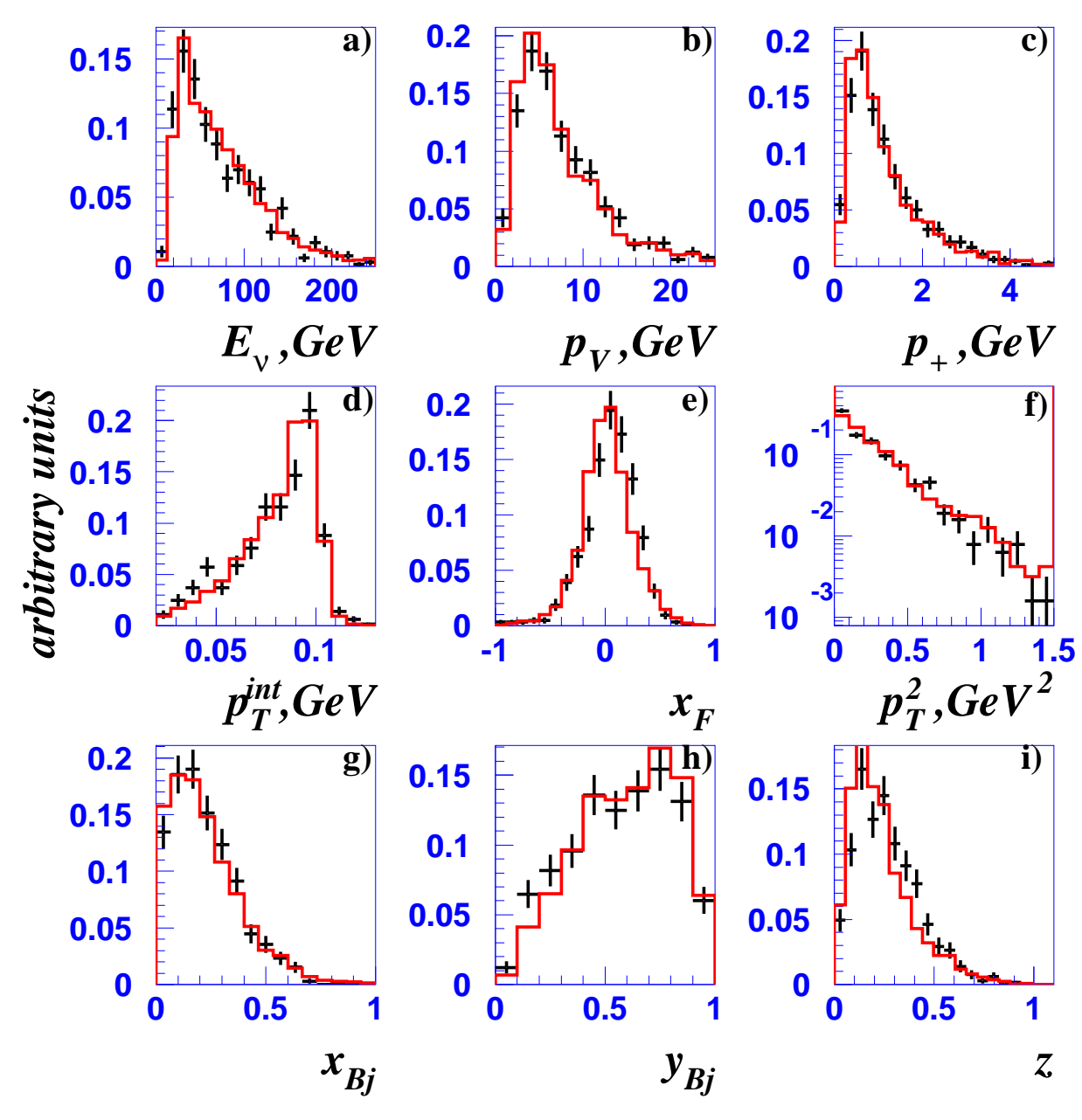

Fig. 3. Comparison of reconstructed kinematic variables for $\nu_{\mu} C C$ events containing identified $\bar{\Lambda}$ hyperons for simulated (histogram) and data (points with error bars) events: a) neutrino energy, b) $\bar{\Lambda}$ momentum, c) momentum of the outgoing $\pi^{+}$ from the $\bar{\Lambda}$ decay, $d$ ) transverse component of the momentum of one of the outgoing charged tracks with respect to the $V^{0}$ direction (internal $\left.p_{T}\right)$, e) $x$-Feynman, $f$ ) square of the transverse component of the $\bar{\Lambda}$ momentum with respect to the hadronic jet direction, g) $x$-Bjorken, h) y-Bjorken, i) fraction of the total hadronic energy carried by the $\bar{\Lambda}$ in the laboratory system.

the detector acceptance and the reconstruction algorithm:

$$
\frac{d N\left(\cos \theta_{i}\right)}{d \cos \theta_{i}}=A\left(\cos \theta_{i}\right)\left(1+\alpha P_{i} \cos \theta_{i}\right)
$$

where $A\left(\cos \theta_{i}\right)$ is the detector acceptance function which also depends on other kinematic variables. The angular distributions in the $\bar{\Lambda}$ case (see Fig. 4) are less distorted than in the case of the $\Lambda$ sample (see e.g. [1]) because of a higher average momentum of $\bar{\Lambda}$ hyperons produced in $\nu_{\mu} \mathrm{CC}$ interactions. This 
is due to baryon number conservation which implies a higher $W^{2}$ threshold for $\bar{\Lambda}$ production in neutrino-nucleon DIS.
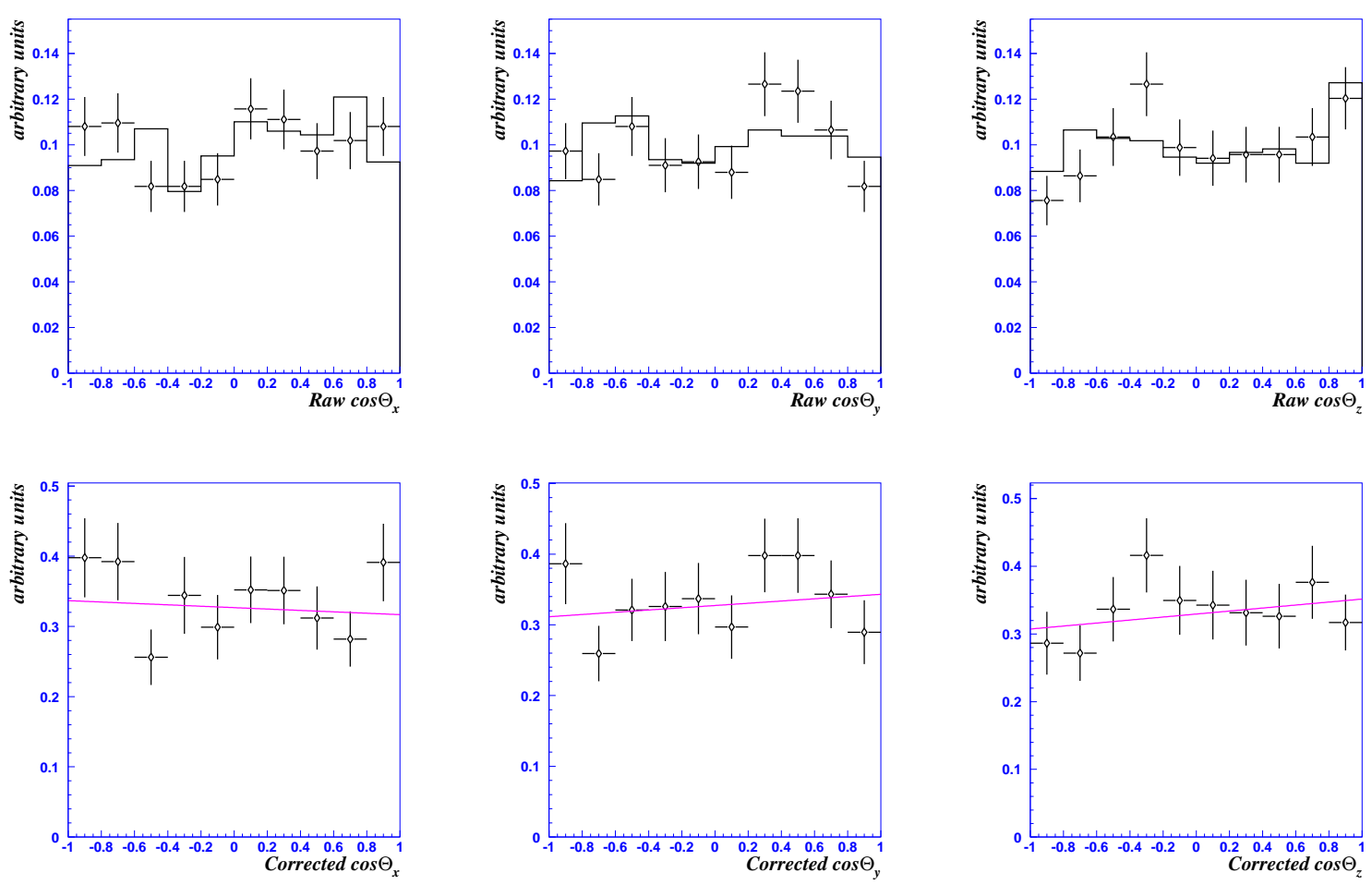

Fig. 4. Top: normalized angular distributions $\left(\cos \theta_{i}\right)$ for $\bar{\Lambda}$ hyperons in reconstructed Monte Carlo events (histogram) and in data (points with error bars). Bottom: experimental angular distributions corrected for detector acceptance and selection effects; the polarization is given by the slope of the corresponding linear fit.

A method which allows the extraction of all three components of the $\Lambda(\bar{\Lambda})$ polarization vector at the same time, taking into account both the detector acceptance and the differences between generated and reconstructed angular variables, has been developed $[1,20]$. The one-dimensional option of this method is used for the analysis presented here because of the low statistics of the $\bar{\Lambda}$ sample.

The results are summarized in Table 1.

\section{Systematic errors}

The following potential sources of systematic errors have been studied (see details in [1]):

- uncertainty in the incoming neutrino energy determination resulting in an 
Table 1

$\bar{\Lambda}$ polarization in $\nu_{\mu} C C$ events (the first error is statistical and the second is systematic).

\begin{tabular}{|c|c|c|c|c|}
\hline \multirow[b]{2}{*}{ Selection } & \multirow[b]{2}{*}{ Entries } & \multicolumn{3}{|c|}{$\bar{\Lambda}$ Polarization } \\
\hline & & $P_{x}$ & $P_{y}$ & $P_{z}$ \\
\hline full sample & 649 & $-0.07 \pm 0.12 \pm 0.09$ & $0.09 \pm 0.13 \pm 0.10$ & $0.10 \pm 0.13 \pm 0.07$ \\
\hline$x_{F}<0$ & 248 & $0.23 \pm 0.20 \pm 0.15$ & $0.04 \pm 0.20 \pm 0.19$ & $-0.08 \pm 0.21 \pm 0.12$ \\
\hline$x_{F}>0$ & 401 & $-0.23 \pm 0.15 \pm 0.08$ & $0.10 \pm 0.17 \pm 0.05$ & $0.25 \pm 0.16 \pm 0.06$ \\
\hline$x_{B j}<0.2$ & 331 & $-0.12 \pm 0.17 \pm 0.08$ & $0.08 \pm 0.18 \pm 0.11$ & $0.01 \pm 0.17 \pm 0.07$ \\
\hline$x_{B j}>0.2$ & 318 & $-0.03 \pm 0.17 \pm 0.14$ & $0.10 \pm 0.18 \pm 0.10$ & $0.20 \pm 0.19 \pm 0.07$ \\
\hline
\end{tabular}

Table 2

Summary of relative systematic errors on the three components of the $\bar{\Lambda}$ polarization vector for the full $\bar{\Lambda}$ sample.

\begin{tabular}{||c|c|c|c|c|c||}
\hline \hline$P_{i}$ & $\begin{array}{c}\nu \text { energy } \\
\text { reconstruction }\end{array}$ & $\begin{array}{c}\text { background } \\
\text { uncertainties }\end{array}$ & $\begin{array}{c}\text { variation of } \\
\text { selection criteria }\end{array}$ & $\begin{array}{c}\bar{\Lambda} \text { spin } \\
\text { precession }\end{array}$ & total \\
\hline$P_{x}$ & $2.9 \cdot 10^{-2}$ & $5.1 \cdot 10^{-2}$ & $6.9 \cdot 10^{-2}$ & $9.1 \cdot 10^{-3}$ & $9.1 \cdot 10^{-2}$ \\
$P_{y}$ & $1.0 \cdot 10^{-1}$ & $9.9 \cdot 10^{-3}$ & $2.1 \cdot 10^{-2}$ & $4.9 \cdot 10^{-3}$ & $1.0 \cdot 10^{-1}$ \\
$P_{z}$ & $7.4 \cdot 10^{-3}$ & $3.7 \cdot 10^{-2}$ & $5.3 \cdot 10^{-2}$ & $3.3 \cdot 10^{-3}$ & $6.5 \cdot 10^{-2}$ \\
\hline \hline
\end{tabular}

uncertainty in the reconstructed W-boson direction and, thus, leading to a poor definition of the "J" reference system in which the $\bar{\Lambda}$ polarization is measured;

- uncertainty in the background rate caused by possible differences between MC and data;

- dependence of the final results on the selection criteria;

- spin precession due to the $\bar{\Lambda}$ travelling through the magnetic field of the detector.

We summarize the results of this study for the full $\bar{\Lambda}$ sample in Table 2 . The maximal deviation with respect to the reference result is used as an estimate of the systematic uncertainty. The overall systematic errors are obtained by adding all the contributions in quadrature ${ }^{2}$. In the $\bar{\Lambda}$ analysis the statistical errors are larger than the systematic errors.

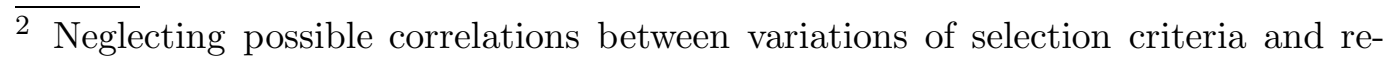
sulting background uncertainties. 


\section{Discussion}

In our previous publication [1] we have reported the measurements of the $\Lambda$ polarization in $\nu_{\mu}$ CC DIS obtained from a sample of 8087 identified $\Lambda \rightarrow \mathrm{p} \pi^{-}$ decays. Let us first briefly recall these results.

For the longitudinal polarization we have found:

- in the target fragmentation region $P_{x}^{\Lambda}=-0.21 \pm 0.04$ (stat) \pm 0.02 (syst). This result is in qualitative agreement with the predictions of the model of negatively polarized $s \bar{s}$ pairs in the nucleon [6].

- in the current fragmentation region $P_{x}^{\Lambda}=-0.09 \pm 0.06$ (stat) \pm 0.03 (syst). This result provides a measure of the spin transfer coefficient $C_{u}^{\Lambda} \approx-P_{x}^{\Lambda}$ (see Eq. (2)).

A significant transverse polarization $P_{y}^{\Lambda}=-0.22 \pm 0.03$ (stat) \pm 0.01 (syst) has also been observed. Its dependence on the transverse momentum of the $\Lambda$ with respect to the hadronic jet direction is in qualitative agreement with the well established behaviour observed in unpolarized hadron-hadron experiments [21].

As shown in Table 1 , the observed $\bar{\Lambda}$ polarization vector for the full data sample is consistent with zero. If we split the $\bar{\Lambda}$ sample into subsamples with $x_{F}>0$ and $x_{F}<0$, we still find results consistent with zero. However, a negative value of $P_{x}^{\bar{\Lambda}}\left(x_{F}>0\right)$, if confirmed, would disagree with the expectations based on calculations [22] performed in the framework of the naive quark model, as well as of the Burkardt-Jaffe [23] and the Bigi-Gustafson-Häkkinen models [24,25]. Similarly, a positive value of the $\bar{\Lambda}$ longitudinal polarization at $x_{F}<0$, if confirmed, would not be consistent with naive expectations in the framework of the model of negatively polarized $s \bar{s}$ pairs in the nucleon [6].

No significant transverse polarization has been found for the $\bar{\Lambda}$ sample.

As a cross-check we have measured the $\Lambda(\bar{\Lambda})$ transverse polarization using a modification of a bias cancelling technique adopted in hadron experiments [26]. We exploit the left-right symmetry of our detector with respect to neutrino beam axis, and separately measure the distributions of Eq. (5) for events with primary vertex on the left $(\mathrm{L})$ or on the right $(\mathrm{R})$ of this axis, replacing $\cos \theta_{y}$ by $-\cos \theta_{y}$ for the right half of the detector: 


$$
\begin{aligned}
& L=\frac{d N_{L}}{d \cos \theta_{y}}=A_{L}\left(\cos \theta_{y}\right)\left(1+\alpha P_{y} \cos \theta_{y}\right) \\
& R=\frac{d N_{R}}{d \cos \theta_{y}}=A_{R}\left(-\cos \theta_{y}\right)\left(1-\alpha P_{y} \cos \theta_{y}\right)
\end{aligned}
$$

Using the fact that $A_{L}\left(\cos \theta_{y}\right)=A_{R}\left(-\cos \theta_{y}\right)$, we define an asymmetry

$$
\epsilon=\frac{L-R}{L+R}=\alpha P_{y} \cos \theta_{y}
$$

which does not depend on the detector acceptance. By fitting $\epsilon$ to a straight line in $\cos \theta_{y}$ we extract the transverse polarization $P_{y}$.

All the measurements of the transverse polarization performed with this method are in a good agreement with the results obtained using our standard method for both $\Lambda[1]$ and $\bar{\Lambda}$ (Table 1 ) samples.

\section{Conclusion}

The results of the $\bar{\Lambda}$ polarization measurements in $\nu_{\mu}$ CC DIS in the NOMAD experiment have been presented. A clean $\bar{\Lambda}$ sample has been selected on the basis of kinematic fits performed on $V^{0}$-like decays. The method used to extract the three components of the $\bar{\Lambda}$ polarization vector accounts for the smearing of the angular variables. While the statistics of the $\bar{\Lambda}$ sample is limited to 649 events, the $\bar{\Lambda}$ polarization has been measured for the first time in a neutrino experiment. The results for the three components of the $\bar{\Lambda}$ polarization vector as measured in the "J" reference system are compatible with zero: $P_{x}=-0.07 \pm 0.12$ (stat) \pm 0.09 (syst), $P_{y}=0.09 \pm 0.13$ (stat) \pm 0.10 (syst), $P_{z}=0.10 \pm 0.13$ (stat) \pm 0.07 (syst). In addition no evidence for the $\bar{\Lambda}$ polarization is found either in the current or in the target fragmentation regions.

More precise measurements of the $\bar{\Lambda}$ polarization in (anti)neutrino interactions could help in clarifying the fragmentation and spin transfer mechanisms of quarks and (anti)quarks into a bound system of antiquarks. Improved experimental results on the $\bar{\Lambda}$ polarization in $\nu(\bar{\nu})$ DIS will be hard to obtain before more intense neutrino beams [27] become available.

\section{Acknowledgements}

We gratefully acknowledge the CERN SPS accelerator and beam-line staff 
for the magnificent performance of the neutrino beam. The experiment was supported by the following funding agencies: Australian Research Council (ARC) and Department of Industry, Science, and Resources (DISR), Australia; Institut National de Physique Nucléaire et Physique des Particules (IN2P3), Commissariat à l'Energie Atomique (CEA), France; Bundesministerium für Bildung und Forschung (BMBF, contract 05 6DO52), Germany; Istituto Nazionale di Fisica Nucleare (INFN), Italy; Joint Institute for Nuclear Research and Institute for Nuclear Research of the Russian Academy of Sciences, Russia; Fonds National Suisse de la Recherche Scientifique, Switzerland; Department of Energy, National Science Foundation (grant PHY-9526278), the Sloan and the Cottrell Foundations, USA.

\section{References}

[1] P.Astier et al., [NOMAD Collaboration], Nucl. Phys. B588 (2000) 3

[2] S.J.Brodsky, J.Ellis, and M.Karliner, Phys. Lett. B206 (1988) 309;

J.Ellis and M.Karliner, Phys. Lett. B213 (1988) 73;

J.Ashman et al., [EMC Collaboration], Phys. Lett. B206 (1988) 364; Nucl. Phys. B328 (1989) 1;

D.Adams et al., [SMC Collaboration], Phys. Rev. D56 (1997) 5330

[3] Review of Particle Properties, Eur. Phys. J. C15 (2000)

[4] D.Buskulic et al., [ALEPH Collaboration], Phys. Lett. B374 (1996) 319;

K.Ackerstaff et al., [OPAL Collaboration], Eur. Phys. J. C2 (1998) 49

[5] A.Airapetian et al., [HERMES Collaboration], DESY-99-151 (1999); hepex/9911017];

M.R.Adams et al., [E665 Collaboration], Eur. Phys. J. C17 (2000) 263, hepex/9911004;

G.Baum et al., [COMPASS Collaboration], CERN-SPSLC-96-14 (1996)

[6] J.Ellis, D.Kharzeev, A.Kotzinian, Z. Phys. C69 (1996) 467

M.Alberg, J.Ellis, D.Kharzeev, Phys. Lett. B356 (1995) 113

[7] B.Ma and J.Soffer, Phys. Rev. Lett. 82 (1999) 2250

[8] B.Ma, I.Schmidt, J.Soffer and J.Yang, Eur. Phys. J. C16 (2000) 657, hep$\mathrm{ph} / 0001259$

[9] B.Ma, I.Schmidt, J.Soffer and J.Yang, Phys. Rev. D62 (2000) 114009, hep$\mathrm{ph} / 0008295$

[10] J.Altegoer et al., [NOMAD Collaboration], Nucl. Instr. and Meth. A404 (1998) 96

[11] M.Anfreville et al., "The drift chambers of the NOMAD experiment", to be submitted to Nucl. Instr. and Meth. 
[12] G.Ingelman, LEPTO version 6.1, "The Lund Monte Carlo for Deep Inelastic Lepton-Nucleon Scattering", TSL-ISV-92-0065 (1992)

G.Ingelman, A.Edin, J.Rathsman, LEPTO version 6.5, Comp. Phys. Comm. 101 (1997) 108, hep-ph/9605286

[13] T.Sjöstrand, "PYTHIA 5.7 and JETSET 7.4: physics and manual", LU-TP-9520 (1995); hep-ph/9508391

T.Sjöstrand, Comp. Phys. Comm 39 (1986) 347, 43 (1987) 367

[14] GEANT : Detector Description and Simulation Tool, CERN Programming Library Long Writeup W5013, GEANT version 3.21

[15] V.Ammosov et al., Nucl. Phys. B162 (1980) 205

[16] D.Allasia et al., Nucl. Phys. B224 (1983) 1

[17] G.T.Jones et al., Z. Phys. C28 (1985) 23

[18] S.Willocq et al., Z. Phys. C53 (1992) 207

[19] D.DeProspo et al., Phys. Rev. D50 (1994) 6691

[20] D.V.Naumov, PhD Thesis, JINR, Dubna, Russia, 2001, in Russian

[21] J.Félix, Mod. Phys. Lett. A14 (1999) 827

[22] A.Kotzinian, A.Bravar, D.von Harrach, Eur. Phys. J. C2 (1998) 329

[23] M.Burkardt and R.L.Jaffe, Phys. Rev. Lett. 70 (1993) 2537

[24] I.Bigi, Nuovo Cim. 41A (1977) 581

[25] G.Gustafson and J.Häkkinen, Phys. Lett. B303 (1993) 350

[26] G.G.Ohlsen and P.W.Keaton, Nucl. Instr. and Meth. 109 (1973) 41

[27] see e.g. CERN Yellow Reports CERN 99-02, CERN 99-12 